\title{
Interactive Image Based Relighting with Physical Light Acquisition
}

\author{
Jianjun Yu, Xubo Yang, and Shuangjiu Xiao \\ Department of Computer Science and Engineering \\ Shanghai Jiao Tong University, Shanghai, China 200240 \\ $\{$ jianjun-yu, yangxubo, xsjiu99\}@sjtu.edu.cn
}

\begin{abstract}
We present an interactive image-based technique to relight real scene with physical light sources acquisition. Firstly, basis images of the real scene are acquired from a fixed viewpoint. Secondly, light direction is estimated and physical light image is captured. Thirdly, measurement image between the reference light image and the novel light image is computed for relighting scene. We demonstrate the technique by interactively relighting a pre-captured real scene using a hand held spotlight projecting light on a white paper.
\end{abstract}

Keywords: Interactive, Image-based Relighting, Physical Light Acquisition, Lighting Sensitive Display.

\section{Introduction}

Image-based relighting(IBL) techniques attract more and more research interest recently. It combines appropriate multiple images and generates novel illuminations in synthesized images [5]. Applications of IBL techniques range from realtime global illumination, digital arts, entertainment computing, light design to mixed reality.

In many applications, virtual scene is required to respond to physical light source or real world environment illuminations. For example, in augmented reality games, if the virtual scene is sensitive to the physical light sources and their change, the game content will integrate closely to the physical environment and enhance the immersiveness of the player. However current IBL techniques usually relight objects or scenes under virtual illuminations or natural/synthetic environments. Few works have addressed the issue of relighting real scenes with physical light source acquisition, and rarely for interaction purpose. Hašan et al[1] presented a fixed view-point interactive relighting. Sun et al[2] described an interactive relighting technique with dynamic BRDFs. Nayer et al proposed a concept of lighting sensitive display, called LSD, which measures the incident illumination and modified its content accordingly[7]. They needed a specially designed camera for acquiring environment light.

In this paper, we present a cheap approach to interactively relighting real scenes based on physical light source acquisition. It can reconstruct realistic appearances of the scenes, and is efficient enough to respond to the hand held 
light changes in real time. Our work is somewhat similar to LSD, however our method for light acquisition and tracking is much easier and cheaper.

\section{Related Works}

Relighting is not a new topic in computer graphics and considerable research has already been done in this field. In article [5], IBL technique was classified into three categories, namely: Reflectance-based, Basis Function-based and Plenoptic Function-based technique.

IBL techniques require no geometry at all. Nimeroff et al[10] used a technique of combining images to relight a scene. Due to the linearity of illumination, objects can be relit by creating a weighted sum of basis images. The weights are calculated using steering functions. Debevec et al 3 address a human face relighting technique, can acquire the reflectance field of a human face and use these measurements to render the face under arbitrary changes in lighting and viewpoint. Masselus et al 4 presents a Free-form Light Stage, a system that captures the reflectance field of an object using a free-moving hand-held light source. And the relighting image is created as a linear combination of basis images with the weights.

Verbeck and Greenberg figure out that "to correctly describe the physical characteristics of light sources, three attributes must be modeled: (1) the light source geometry, (2) the emitted spectral distribution, and (3) the luminous intensity distribution". While these attributes may be necessary and sufficient, they rarely lead to useful models of complex three-dimensional light sources. The problem is common to all point source models: the geometries and photometric distributions of most physical light sources are simply too complicated to describe accurately. The field-theoretic approach [9] [6] that can be used to model arbitrarily complex three-dimensional light sources. Goesele at el 8 develop a method for dealing with complex light sources from acquisition to global illumination rendering by measuring real-world light sources using an optical filters.

\section{Data Acquisition}

To obtain basis data for relighting, Debevec et al describes a series of Light Stages, Masselus et al 4 represent a Freeform Stage and use four diffuse spheres to estimate light directions. We have designed equipment for data acquisition. Our setup of acquisition system (see Fig. 1) is a computer controlled semicircle arm with reference light sources attached to it. We use a Nikon D80 digital camera for acquiring data and 8 Watt white LED as point light sources. The subject is placed in the center of the stage and illuminated by the controlled light sources. For each light source position an image is captured. This process is repeated until the entire sphere of incident illumination is sampled at some resolution. Fig. 1 also displays several images, from the full set of images, illuminated from different directions. 

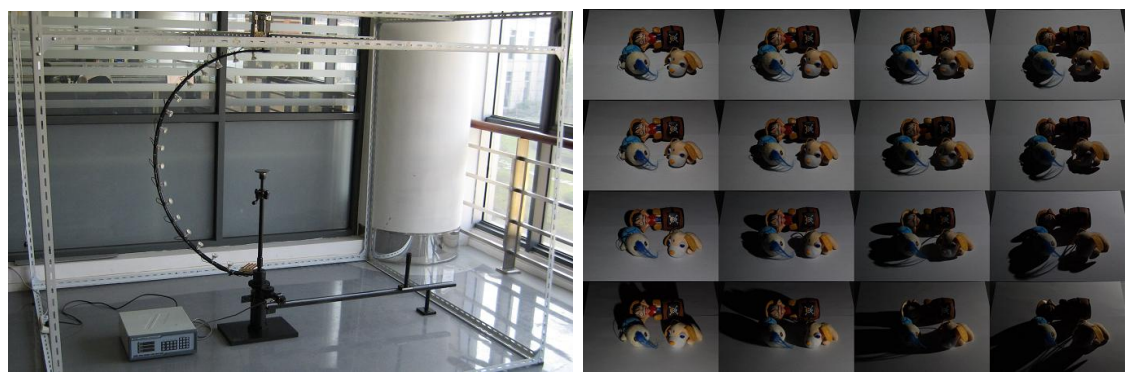

Fig. 1. Light stage and Basis images set

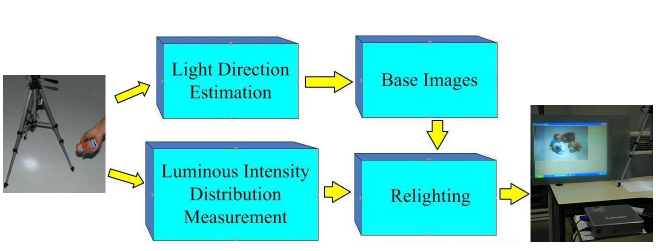

(a)

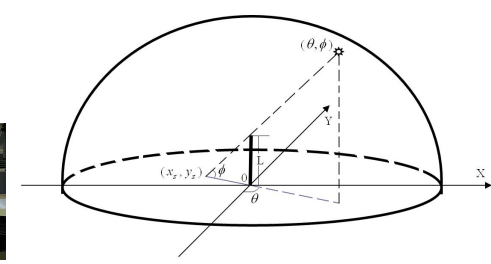

(b)

Fig. 2. System overview and equipment \& Light direction estimation

\section{Interactive Relighting}

We use a webcam camera to capture the physical light sources luminous intensity distribution. Also we use a pole on the paper for estimating the light direction. Then we employ an image-based relighting method to relight the scene under arbitrary physical illumination. The main modules of our system is shown in Fig. 2(a).

\subsection{Light Direction Estimation}

We detect the shadow of pole by using Canny edge detector. The direction of light can be computed with the two vertices of the shadow and the position of the pole. Fig. 2(b) and Equation 1 show how to estimate the light direction.

$$
\begin{aligned}
\theta & =\left\{\begin{array}{l}
\pi-\arctan \left(x_{s} / y_{s}\right) \text { if } x_{s} \geq 0 \\
\pi+\arctan \left(x_{s} / y_{s}\right) \text { if } x_{s}<0
\end{array}\right. \\
\phi & =\arctan \left(L / \sqrt{x_{s}^{2}+y_{s}^{2}}\right)
\end{aligned}
$$

\subsection{Luminous Intensity Distribution Measurement}

Theoretical Framework. We first introduce the mathematical notation used throughout this document. This notation is summarized in Table 1. 
Table 1. Mathematical Notation

\begin{tabular}{ll}
\hline Symbol & Meaning \\
\hline$L_{d i}(x, y)$ & reference light source radiance passing through pixel $(x, y)$ from di direction \\
$\hat{L}_{d i}(x, y)$ & novel light source radiance passing through pixel $(x, y)$ from $d i$ direction \\
$I_{L, d i}(x, y)$ & irradiance caused by $L_{d i}(x, y)$ on diffuse plane \\
$\hat{I}_{L, d i}(x, y)$ & novel irradiance caused by $\hat{L}_{d i}(x, y)$ on diffuse plane \\
$I_{O, d i}(x, y)$ & irradiance caused by $L_{d i}(x, y)$ on object \\
$\hat{I}_{O, d i}(x, y)$ & novel irradiance caused by $\hat{L}_{d i}(x, y)$ on object \\
$L T_{d i}(x, y)$ & diffuse plane's basis function at pixel $(x, y)$ \\
$O T_{O, d i}(x, y)$ & object's basis function at pixel $(x, y)$ under $L_{d i}(x, y)$ \\
$M_{L, d i}(x, y)$ & measurement image between reference and novel light source at pixel $(x, y)$ \\
\hline
\end{tabular}

According to Levin's Field Theory [9], which can accurately predict the direct illuminance at any point on any surface anywhere within the surrounding three dimensional space without requiring any knowledge of the geometry of the source or its distance from the surface being illuminated, the light distribution in region of interesting can be described as Fig 3 . So we can represent a light source image by Equation 2 .

$$
\begin{gathered}
I_{L, d i}(x, y)=L T_{d i}(x, y) \cdot L_{d i}(x, y) \\
I_{O, d i}(x, y)=O T_{O, d i}(x, y) \cdot L_{d i}(x, y)
\end{gathered}
$$

So, Equation 3 describes object image with different direction incident illuminations:

$$
I_{O}(x, y)=\sum_{d i=1}^{N} O T_{O, d i}(x, y) \cdot L_{d i}(x, y)
$$

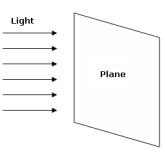

(a)

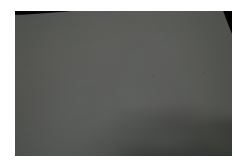

(b)

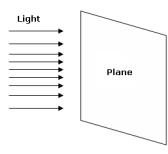

(c)

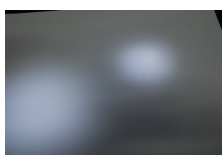

(d)

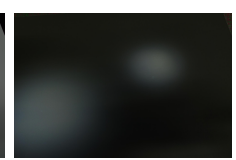

(e)

Fig. 3. Reference and Physical Light. (a),(c): show the distribution of reference incident and physical incident; (b),(d): show the distribution of incident in light image;(e)shows measurement image for visualization purposes.

\subsection{Image-Based Relighting}

Relighting is the process of rendering the captured reflection data as illuminated by some novel lighting conditions. Ray Tracing method traces rays of light from the eye back through the image plane into the scene. It assumes that there is one backward light ray belonging to each pixel and the color of the pixel is decided by the light ray. So, if we can measure the difference between the reference light 
image and the novel light image, then we can relight the object under novel light source. While we denote novel physical light source using $\hat{L}_{d i}(x, y)$ and an object image under $\hat{L}_{d i}(x, y)$ by $\hat{I}_{O}(x, y)$. We can get our relighting equation (see Equation 4) by employing the ray tracing idea. Then we get Equation 4

$$
\begin{aligned}
\hat{I}_{O}(x, y) & =\sum_{d i=1}^{\hat{N}} O T_{O, d i}(x, y) \cdot \frac{\hat{I}_{L, d i}(x, y)}{I_{L, d i}(x, y)} \cdot L_{d i}(x, y) \\
& =\sum_{d i=1}^{\hat{N}} O T_{O, d i}(x, y) \cdot L_{d i}(x, y) \cdot M_{L, d i}(x, y)
\end{aligned}
$$

Equation 4 shows that we can use the basis images and measurement weights to relight object under novel light sources. Then, a measurement image (see Fig (3) between the reference light image and physical light image is calculated for relighting. In order to remove the effects of pole's shadow, we use an interpolation algorithm for compensation.

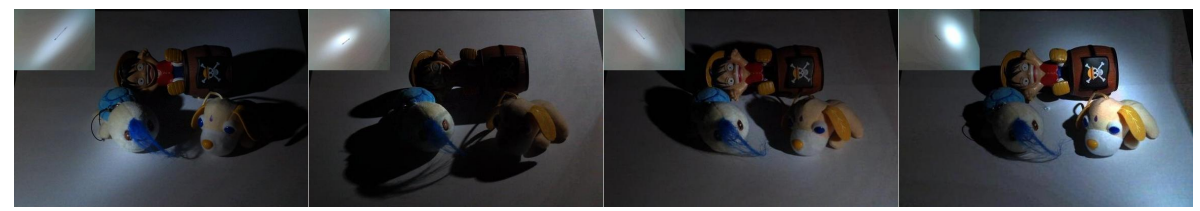

Fig. 4. System outputs with different illuminations

\section{Experiments}

In our experiment, we achieve real time performance using $2.8 \mathrm{GHz} \mathrm{CPU}$ and the appearance of the scene including shadows and specularities is represented with the position of the hand held spotlight. The major advantage of our method is that it can handle successfully image-based relighting under interactive physical light illumination using a simple and cheap configuration. We sampled a scene and relight it using our technique. Four relit images are shown in Fig, 4 , where the small window at the top-left corner of each images shows the corresponding projected light on the white paper. We also use a paper with texture instead of the white paper which the hand held spotlight project on. The interesting result shows our light acquisition method is insensitive to the background. It means that we can implement system in more nature environment.

\section{Conclusion and Future Work}

In this paper we have presented a simple and cheap interactive image-based technique to relight real world scenes interactively with physical light acquisition. Our solution makes it possible to relight surfaces with complex material properties under interactive light illuminations using simple equipments. 
Our algorithm may be improved in several ways. Since we only handled the linear relationship, light acquisition can be refined by a more efficient manner. To achieve more natural interactions, we can improve on the compensation technique as well.

Acknowledgments. This work is sponsored by 863 National High Technology R\&D Program of China (No. 2006AA01Z307) and National Natural Science Foundation of China (No. 60403044) and partially by Omron company.

\section{References}

1. Hašan, M., Pellacini, F., Bala, K.: Direct-to-indirect transfer for cinematic relighting. In: ACM SIGGRAPH, pp. 1089-1097. ACM Press, New York (2006)

2. Sun, X., Zhou, K., Chen, Y.Y., Lin, S., Shi, J.Y., Guo, B.N.: Interactive Relighting with Dynamic BRDFs. In: ACM SIGGRAPH. ACM Press, New York (2007)

3. Debevec, P., Hawkins, T., Tchou, C., Duiker, H.P., Sarokin, W., Sagar, M.: Acquiring the Reflectance Field of a Human Face. In: ACM SIGGRAPH, pp. 145-156. ACM Press, New York (2000)

4. Masselus, V., Dutre, P., Anrys, F.: The Free-form Light Stage. In: Eurographics Workshop on Rendering, pp. 26-28 (2002)

5. Choudhury, B., Chandran, S.: A Survey of Image-based Relighting Techniques. In: The Proceedings of the International Conference on Computer Graphics Theory and Applications (GRAPP) (2006)

6. Ashdown, I.: Near-Field Photometry: Measuring and Modeling Complex 3-D Light Sources. In: ACM SIGGRAPH '95 Course Notes - Realistic Input for Realistic Images, pp. 1-15 (1995), citeseer.ist.psu.edu/ashdown95nearfield.html

7. Nayar, S.K., Belhumeur, P.N., Boult, T.E.: Lighting Sensitive Display. In: Proceedings of ACM SIGGRAPH, Technical Sketch, vol. 23(4), pp. 963-979 (October 2004)

8. Goesele, M., Granierd, X., Heidrich, W., Seidel, H.P.: Accurate Light Source Acquisition and Rendering. ACM Transactions on Graphics 22(3), 621-630 (2003)

9. Levin, R.E.: Photo metric characteristics of light-controlling apparatus. Illuminating Engineering 66(4), 205-215 (1971)

10. Nimeroff, J., Simoncelli, E., Dorsey, J.: Efficient Rerendering of Naturally Illuminated Environments. IEEE Computer Graphics and Applications (1994) 